\title{
Ramón Reichert
}

\section{„Volunteering“"und,„Bürgerarbeit“"in Gemeinwohl- Konzepten

\author{
Überlegungen zur unbezahlten Arbeit
}

\begin{abstract}
Im Dritten Sektor werden allgemein bisher nicht erschlossene Beschäftigungsmöglichkeiten vermutet Schlagworte wie „Neues Ehrenamt“, „Bürgerarbeit“" oder „Volunteering“ kennzeichnen die Suche nach „brachliegendem“ Humankapital. Als Musterbeispiel des organisierten „Volunteering“ gelten die USA. Aber auch innerhalb der Europäischen Union wurde im letzten Jahrzehnt die Bewirtschaftung des "Engagementpotentials" als arbeitsmarktpolitisches Regulativ forciert. Am Beispiel der aktuellen Diskussionen über den Beitrag zivilgesellschaftlicher Arbeit zur Erhaltung der demokratischen Ordnung wird untersucht, wie die Selbstregulationsfähigkeit von Individuen und Gruppen mit ökonomischer Profitmaximierung und gesellschaftspolitischen Zielen verknüpft werden. Der Beitrag geht davon aus, dass in den Debatten um den Stellenwert sozialer Anerkennung von unbezahlter Arbeit gleichermaßen ein neues Subjekt der Arbeit konstruiert wurde, darin wirtschaftlicher Wohlstand und persönliches Wohlsein miteinander gekoppelt sein sollen. Im Zentrum der Untersuchung steht die Untersuchung der Genese und der argumentativen Rechtfertigung der Beziehungen zwischen dem Abbau sozialstaatlicher Leistungen und dem zunehmenden Appell an „Eigenverantwortung“" und „Selbstsorge“.
\end{abstract}

\section{$1 \quad$ Von der passiven zur aktiven Dienstleistung}

In den gegenwärtigen Konzepten zur Aufwertung der ehrenamtlichen Tätigkeit soll die entscheidende Wende vom alten karitativen Ehrenamt zum aktiven, freiwilligen Engagement als soziale Innovation vollzogen werden. Geht es nach dem Konzept des „Neuen Ehrenamtes" (exemplarisch Olk, 1998), dann hat das Image der passiven, abhängigen und selbstlosen Dienstleistung ausgedient. Mit dem Gegensatzbegriff des aktiven, unabhängigen und individualistischen „Service” gilt es, die als ,zeitgemäß” bestimmte Dienstleistung endgültig von ihrer Geschichte der Subsistenz, der materiellen Lebensgrundlage sowie ihrer Subordination, der sozialen Unterordnung zu lösen.

Vor der ersten industriellen Revolution war die Begleitung von Armen, Kranken, Behinderten und Betagten fast ausschliesslich eine Angelegenheit der kirchlichen Gemeinde. In den Industrienationen des 19. Jahrhundert wurde die soziale Dienstleistung zunehmend in Form von Armengesetzen, Regelungen der Arbeitszeit, Schulpflicht und Verbot der Kinderarbeit zum Gegenstand allgemein gültiger Gesetzgebung. Doch die Angst der angesehenen Bürger vor revolutionären Aktionen der Arbeiterbewegung am Höhepunkt der ersten 
Industrialisierung blieb unbeschwichtigt und führte zum Spleen, gemeinnützige Gesellschaften zu gründen. Die Einrichtung von gemeinnützigen Vereinen korrespondierte mit der „Staatsfeindlichkeit“ der besitzenden Eliten, die die Ausweitung der Staatstätigkeit im Bereich der Infrastruktur und der Sozialpolitik - als Ergebnis des Kampfes der sozialistischen Arbeiterbewegung seit dem späten 19. Jahrhundert - stets nur widerwillig hingenommen bzw. politische Kräfte unterstützt haben, die - wie in Deutschland nach der Novemberrevolution 1918/1919 - auch die einfachsten demokratischen Errungenschaften der Revolution (wie die parlamentarische Republik und den Acht-Stunden-Tag) revidieren wollten.

Die christlich-karitative Nächstenliebe bildete die moralische Ermächtigung, wie Nietzsche treffend analysiert, sich das auserwählte Opfer sozial bewahrend zur „Ordinierung einer kleinen Freude” zurecht zu machen: „Das Glück der ,kleinsten Überlegenheit“ - wie es alles Wohltun, Nützen, Helfen, Auszeichnen mit sich bringt - ist das reichlichste Trostmittel” der „hülfreichen und wohlthätigen Menschen” (Nietzsche 1994, 876). Frauen privilegierter sozialer Schichten gründeten ihre eigenen Vereine. Ziel der Vereinigungen der Bürgersfrauen war es, die „,armen Frauen“ zu ähnlich „,guten Hausfrauen“ zu erziehen. Die unbezahlten Sozialisierungen der „Hilfsbedürftigen“ galten nicht als Arbeit, sondern nur als eine erweiterte private Haushaltsaufgabe.

Neuerdings korrespondiert der Antietatismus auch der Interessenlage jener Mittelschichten, die die Solidarität mit der „underclass“ (dieser Block trug im Wesentlichen die Reformpolitik seit den späten 60er-Jahren) aufgekündigt hat, universalistische Gesellschaftspolitik mehr und mehr ablehnt und stattdessen private Dienste für ihre Bildung, Gesundheits- und Altersversorgung sowie für Kultur und Sicherheit bevorzugt.

Als Ausgangspunkt der Professionalisierung der unbezahlten Dienstleistung gilt die Seinsgewissheit einer unaufhaltsamen Knappheit bezahlter Erwerbsarbeit. Die Annahme, dass bezahlte Arbeit ,ausgehe”, bezieht sich auf sozial anerkannte Erwerbs-oder Lohnarbeit, vorwiegend im industriellen Sektor. Für die Zukunft wird ein größerer Zwischensektor genossenschaftlicher, kommunaler Tätigkeit als Sektor für „Gemeinwirtschaft” angenommen, darin gemeinnützige Arbeit nicht über Geld entlohnt, sondern über Zeit verrechnet wird. Wer aktiv ist, muss Zeit besitzen und erübrigen können. André Gorz sah in seiner 1989 verfassten „Kritik der ökonomischen Vernunft“eine neue „Dienstbotengesellschaft“ entstehen, in welcher die mit den entsprechenden Vermögen ausgestatteten Bürger die persönlichen Dienstleistungen der von Erwerbsarbeitslosigkeit Betroffenen in Anspruch nehmen, und dieser sozial deklassierte Teil der Bevölkerung darauf angewiesen ist, sich in die Abhängigkeit persönlicher Dienstleistungsverhältnisse zu begeben (Gorz 1989, 218ff). Zahlreiche Studien über den „Dritten Sektor“ zeigen, dass sich die sozial Engagierten überwiegend aus Erwerbstätigen mit hohem Einkommen, guter Ausbildung und mit Familie zusammensetzen, die das nötige „soziale Kapital“ mitbringen: Netzwerke und Kommunikationsfähigkeiten. Zivilgesellschaftliche Arbeit wird von den Gewinnern der Wohlfahrt geleistet, von im Zeitwohlstand lebenden „Vollzeitbürgern”. Sie fungieren als strukturkonservative „(Ehren)Beamte” des Gemeinwesens, die ihre armen Schäfchen in der Herde hüten - Diener der Moral für moralisch Bediente.

In den letzten Jahren rückte der Arbeitsbegriff in das Zentrum der Demokratietheorien des amerikanischen Kommunitarismus, dessen pragmatisch orientierte Repräsentanten Amitai Etzioni, Benjamin Barber und Robert Belah und ihre Schriften auch in Deutschland breite Beachtung fanden (Reese-Schäfer 1996, 4; Roth 1995, 44-53). In der Graswurzeldemokratie der „Aktivbürger“ hat der was zu sagen, der an endlosen Versammlungen teilnimmt. Darin 
sind Bürgerrechte nicht aus Besitz, Eigentum, Erbe oder Privilegien ableitbar, sondern müssen in einer andauernden Partizipation stetig erneuert werden.

Während der „Aktivbürger” auf der Suche nach sozialer Anerkennung mit dem moralischen Verdienst zufrieden gestellt ist, investiert der Staat als Betrieb in weitere outsourcingMaßnahmen zur Professionalisierung des ehrenamtlichen Sektors. Im Schatten dieses hegemonialen Diskurses mit seiner Rhetorik von „Befreiung” und „Selbstfindung” übernimmt der „Volunteer” jedoch soziale Kosten (z.B. Umweltbelastung) und entlastet als - zuvor brachliegende - Ressource lebendiger Arbeit staatliche Wohlfahrtsleistungen. Wie häusliche Arbeit schafft auch volunteering Wohlfahrt, ist aber nicht bezahlte Berufstätigkeit.

Auf der Ebene von soziologischen Analysen wird oft das „Ende der Arbeit“als Epochenbruch postuliert, und der „Zukunft der Arbeit“ gegenübergestellt, die notwendig und unwiderruflich aus dem Dritten Sektor hervorgehen soll. Beispiele hiefür sind zahlreiche gesellschaftspolitische Prognosen zur „Zukunft der Arbeit“ u.a. von Jeremy Rifkin („Das Ende der Arbeit und ihre Zukunft“), Ulrich Beck u.a. in „Die Zukunft von Arbeit und Demokratie“ und in „Schöne neue Arbeitswelt“, Richard Sennett (,Der flexible Mensch“) sowie André Gorz (,Arbeit zwischen Misere und Utopie“). Am dramatischsten hat in jüngster Zeit der US-Autor Jeremy Rifkin vom „Ende der Arbeit“ gesprochen. „20 Prozent der arbeitsfähigen Bevölkerung würden im kommenden Jahrhundert ausreichen, um die Weltwirtschaft in Schwung zu halten“, verkündet Jeremy Rifkin in seinem Buch „Das Ende der Arbeit und ihre Zukunft“, ,die unteren 80 Prozent werden gewaltige Probleme bekommen“ (Rifkin 1997, 24).

Folgt man den Prognosen von Rifkin, so ist damit zu rechnen, dass die künftigen gesellschaftlichen Strukturen der genannten „Informationsgesellschaft“ von wachsender Erwerbsarbeitslosigkeit und einem neuartigen gesellschaftlichen Segmentierungsprozess geprägt sein werden. Ein Segmentierungsprozess, der ein Ende der fordistischen Massenbeschäftigung zu Gunsten der Ausbreitung von informellen Tätigkeiten und Diensten verfolgt. Rifkins Konzept der „Zukunft der Arbeit“ läuft auf eine Zweiteilung von wirtschaftlichem Leben einerseits und andererseits dem „Dritten Sektor“ ehrenamtlicher und kultureller Aktivität hinaus.

\section{Politische Moralisierung des volunteering in den USA}

In den Studien über neue Formen der Dienstleistung gelten die USA als Musterbeispiel des organisierten volunteering (Beck 1999b, 141). Nach der Statistik des „Organisation Independent Sector" leisten rund die Hälfte aller erwachsenen AmerikanerInnen durchschnittlich 4,2 Stunden pro Woche regelmäßig Arbeitseinsätze als volunteer. Diese kollektive Arbeitsleistung entspricht einem Geldwert von 201,5 Milliarden Dollar(Wuthnow, 1991). In Anbetracht dieser gewaltigen Ressource von Gratisarbeit fand im Frühjahr 1997 in den USA unter dem Titel „The President's Summit for America's Future” eine Medienshow zum Thema volunteering statt. In seiner big speech an die Nation legte Präsident Clinton den AmerikanerInnen big citizenship ans Herz, als Alternative zum vielgeschmähten big government. Der Begriff citizenship umfasst das Recht und die Pflicht, sich als Bürger in das öffentliche Leben einzumischen. Gemeinsame Werte verschaffen nationale Identität und Zugehörigkeit - der Leitspruch ,volunteering is a set of values [...] to be part of something bigger” (Kramer 1981, 27) wurde zum Erweckungsruf für das spirituelle Erlebnis des ,inneren Vaterlandes” 
auserkoren. Mit diesem Appell verdeutlicht Clinton die amerikanische Tradition, soziale Reformen als patriotische Bürgerpflicht zu gebieten. In unmittelbarer Bezugnahme auf John F. Kennedys Anfang der Sechzigerjahre propagierten Motto „Ask not what your country can do for you, but ask instead what you can do for your country" zur Einschwörung der Gemeinschaft, die als ,,soziale Reform“"nach seinem Tod von Lyndon B. Johnson fortgeführt wurde, soll Gratisarbeit die Lücke füllen, die sich zwischen der staatlichen Fürsorge und dem freien Markt auftue.

Voluntarismus gilt traditionell als der amerikanische Ersatz für eine monetär umverteilende Sozialpolitik. Die starke Tradition der Philanthropie sowie das Fehlen einer Staatskirche hatten die Entstehung privater Wohlfahrtsorganisationen begünstigt. Erst das Massenelend der Depression zwang zum Umdenken: Präsident Roosevelt schuf mit dem New Deal ein (im Vergleich mit Europa stark verspätetes) staatliches Sozialnetz. Der private Sektor wurde damit nicht obsolet: Die öffentliche Verantwortung wird zwischen dem staatlichen Bereich des public sector einerseits und dem privaten Bereich des independent oder non-profit sector andererseits geteilt (Belahs, u.a. 1985). Der unabhängige Sektor ist seit den 60er Jahren rasant gewachsen: mehr als ein Drittel der Arbeit in den Bereichen Religion, Soziales, Kultur, Gesundheit und Bildung wird von volunteers geleistet. Das Verhältnis zwischen der und der privaten Wohlfahrt ist komplex - nicht nur weil die Non-profit-Organisationen als Folge ihres steuerfreien Status indirekt subventioniert werden. Seit den 60er Jahren verschwimmen die Grenzen zunehmend, denn um Kosten zu sparen, übertragen öffentliche Einrichtungen wie Spitäler, Gefängnisse oder Erholungsparks immer grössere Teile ihres Betriebs an private Non-profit-Organisationen, die damit ihrerseits zunehmend von öffentlichen Geldern abhängig werden.

Auch Ronald Reagan rief die Amerikaner zum volunteering auf - nun allerdings in einer Umkehrung von Johnsons ,war on poverty“: Die Amerikaner müssten wieder freiwillig tun, was sie sich vom Staat hätten abnehmen lassen (Ellis/Noyes, 1990, 17f.). Der Hintergrund von Reagans Appell bestand nicht in einem Ausbau des Sozialstaats, sondern in massiven Kürzungen des Sozialbudgets.

Im Vergleich zu den früheren Jahren ist das freiwillige soziale Engagement noch weiter gewachsen und hat sich in den USA auch stabilisiert. Im Vergleich zu europäischen Ländern lässt sich zwar in den USA der klassische Typus des Engagierten (berufstätig, relativ gutes Einkommen und hoher Bildungsstatus) nachweisen, dennoch streut das Engagement stärker über Berufs- und Statusgruppen, was wiederum einen Hinweis auf die starke Verankerung der Freiwilligkeitskultur gibt. Einerseits erstreckt sich in den USA das volunteering über viel mehr Sektoren als beispielsweise in Deutschland, das reicht von der Telefonberatung über Klienten-Lobbying bis zur aktiven Gemeinwesenarbeit. Zweitens hat sich in den USA das volunteering stärker professionalisiert. Die Professionalisierung macht sich nicht nur in den Praktiken systematischer Rekrutierung, sondern auch institutionell bemerkbar. Anders als in der Bundesrepublik existieren in den USA auf lokaler, regionaler und nationaler Ebene eigene Volunteer-Bureaus. Diese Büros betreiben eine eigene Marktforschung, versuchen Profile und Interessierte zusammenzubringen und betreiben public relations. Zugleich organisieren und finanzieren US-Unternehmen über ihre Arbeitnehmer das volunteering mit. Dieses social sponsoring zählt zur corporate identity, auf den pay rolls werden Lohnabzüge angespart, mit denen gemeinnützige Aufgaben finanziert werden. Die Beteiligung der Unternehmen an gemeinnützigen Aktionen wie beispielsweise den days of caring, an denen Arbeitnehmer für das Gemeinwohl freigestellt werden, werden schließlich veröffentlicht und 
als agenda setting marketingwirksam. Obwohl auch in Großbritannien der Sektor der institutionalisierten sozialen Dienste breiter ist als etwa in Deutschland und auch den Bereich der Rechtsberatung und des Bildungswesen umfasst, hat sich bislang allerdings keine den USA vergleichbare Professionalisierung ehrenamtlicher Arbeit herausgebildet.

Das Räsonnieren über Potentiale „,bürgerschaftlichen Engagements“ entspringt der Diskussion um Methoden der Verwaltungsmodernisierung. Auf kommunaler Ebene werden BürgerInnen im Zuge der Verwaltungsmodernisierung zu „Kunden“ umgewidmet, denen man möglichst effizient die gewünschten Leistungen anbieten müsse. In verschiedenen sozial-und wirtschaftswissenschaftlichen Disziplinen sind demzufolge die normativen und kulturellen Grundlagen politischer und wirtschaftlicher Institutionen wieder zum Thema geworden. In der Kommunitarismusdiskussion - wie auch in den Debatten um den Neuen Institutionalismus - stehen Begriffe wie social capital (Coleman, 1988; Helliwell, 1996) oder civicness (Putnam, 1994) paradigmatisch für diese Entwicklung.

Eine richtungweisende moraltheoretische wie auch sozialpolitische Einbettung erhielt das volunteering in dem von der „Communitarian Platform“ am 18. November 1991 veröffentlichten kommunitaristischen Programm (Etzioni 1995, 282-299). Dieses Programm wurde als repräsentativ für das Denken der Kommunitarier ausgewiesen. In der Erstversion von Amitai Etzioni verfasst, dann von Mary Ann Glendon und William Galston modifiziert, stellt es letztlich das Ergebnis eines damals veranstalteten Kongresses dar. Etzioni selbst schreibt hierzu, dass dieses Programm "unseren Ansatz zusammenfasste und unsere Grundpositionen auflistete. (...) Es wurde von siebzig führenden Persönlichkeiten aus den verschiedensten Bereichen unterschrieben, was seine öffentliche Akzeptanz erhöhte.” (Etzioni 1995, 21) Im Kapitel über „SSoziale Gerechtigkeit““wird von der „Idee der Reziprozität“" gesprochen: „Jedes Mitglied der Gemeinschaft ist allen etwas schuldig, die Gemeinschaft schuldet jedem ihrer Mitglieder etwas. Gerechtigkeit erfordert verantwortungsbewusste Individuen in einer verantwortlichen Gemeinschaft.“ (Etzioni 1995, 289) Die Kommunitarier setzen auf die traditionelle Sozialversorgung der USA, auf die Initiativen ,,von unten“, auf lokale Aktivitäten, Selbsthilfegruppen, Kirchengemeinden. Ihnen geht es weniger um die staatliche Übernahme sozialstaatlicher Verantwortung, sondern mehr um die Kritik am bürokratischen Wohlfahrtsstaat. Allerdings wird auch davon ausgegangen, dass der Staat bzw. die Gemeinschaft die Pflicht habe, "die Grundbedürfnisse derer zu decken, die wirklich nicht für sich selbst sorgen können" (Etzioni 1995, 295) Die Kommunitaristen plädieren für eine gemeinschaftliche Für- und Vorsorge, die, basierend auf dem Prinzip der Subsidiarität, die sozialstaatliche Versorgung ergänzt.

Volunteering wird von den Kommunitaristen ursprünglich der Instanz des moralischen Gewissens unterstellt. Um volunteering zum kollektiven Gewissen aufzubauen, muss es zum Gegenstand der moralischen Bildung und folglich der nationalen Erziehung, der ,wechselseitigen Einfühlung und Respekt“" (Barber 1994, 214), erhoben werden. Um diesen kollektiven „Bürgersinn“als konstantes Motiv herzustellen, setzt Etzioni in seinem Aufsatz „Über den Eigennutz hinaus“ auf intermediäre Institutionen der Zivilgesellschaft: „Wird er zu Hause nahe gebracht, in der Schule gefördert, in den Medien herausgestellt, von freiwilligen Vereinigungen unterstützt und von führenden Persönlichkeit, offiziell ‘ verkündet, so fühlen sich die Bürger eines Landes verpflichtet, etwas für das Wohl ihrer Gemeinschaft zu tun.“ (Etzioni 1993, 121)

In den Gemeinwohl-Konzepten wird generell von einem voluntaristischen Bürgerbegriff ausgegangen, darin der volunteer durch seine Tätigkeit seine „persönlichen Bedürfnisse“ 
befriedigen möchte, „Spaß“ haben und alltäglich mit „,neuer Begeisterung“ an das Werk gehen möchte (Barber 1994). Für uns alle, postulieren tonangebende sozialforschende Regierungsberater wie Benjamin Barber, Amitai Etzioni oder Robert Belah, bedeute dieser Wandel die Chance für mehr Persönlichkeit, darin Lebensgeschichte und Erwerbsbiografie zur Gratis-Ausbildung gemeinschaftlicher Werte verschmelzen sollen: Sozialkompetenz, Netzwerkfähigkeiten, Teamgeist, Gruppenkultur etc. (Etzioni 1996, 403) Im unmittelbaren Lebensumfeld erfährt der Volunteer empowerment, jenes „Erlebnis“, etwas bewirken zu können. In diesem Sinn lässt sich die grosse Bereitschaft zum volunteering auch als Kehrseite der Entpolitisierung der amerikanischen Gesellschaft interpretieren.

Die Bildung von moralischen Normen sind für die politische Praxis vorteilhaft, sie senken Transaktionskosten, juristische, administrative und polizeiliche Verfahrensweisen und steigern die Produktivität des Volkseinkommens. Weiter verhilft die Annahme eines auf einer moralischen Verpflichtung basierenden „Bürgersinns“der Sozialökonomie zu besseren Voraussagen.

In Deutschland dominiert gegenwärtig die sozialwissenschaftliche Debatte um „Bürgerschaftliches Engagement“ und „Bürgerarbeit“ der politikberatende Soziologe Ulrich Beck, der oft ausgehend vom Leitbild des US-amerikanischen Kommunitarismus in zahllosen Publikationen die zivilgesellschaftliche Arbeit als unverzichtbaren Beitrag zur Erhaltung der demokratischen Ordnung propagiert und dadurch den Hegemonieanspruch der Sozialwissenschaften für die politische Anwendung, d.h. die ,soziale Frage“, verdeutlicht. Der Begriff der „Verinnerlichung“ nimmt in (seinen) Schriften ebenfalls einen zentralen Stellenwert ein, er bezeichnet „Verinnerlichung“als „Schlüsselerlebnis, das die gesellschaftliche Wirklichkeit politischer Freiheitsräume individuell erfahrbar macht." (Beck 1998, 203).

Liest man die Ausführungen renommierter Politikberater wie Amitai Etzioni oder Ulrich Beck gegen den Strich, bringen sie die Kernthese Antonio Gramscis betreffend die Funktionsweise der Zivilgesellschaft als hegemoniale Konsensgemeinschaft auf den Punkt. Gramsci definiert bekanntlich Zivilgesellschaft als jenen Teil des Sozialen, in dem der ,integrale Staat" den Konsens der Zivilbürger mit den bürgerlichen Werten organisiert und so die Hegemonie der herrschenden moralischen Disziplin sichert (Demirovic 1997, 165-182). Der Einsatz herrschaftlicher Kompetenzen bleibt ineffektiv und jedenfalls ineffizient, wenn er nicht vom „Legitimitätsglauben“ der Adressaten gestützt wird. Dieser erst sichert, in Max Webers Formulierung, die Chance des Gehorsams in der Weise, „dass das Handeln des Gehorchenden im Wesentlichen so abläuft, als ob er den Inhalt des Befehls um dessen selbst willen zur Maxime seines Verhaltens gemacht habe“ (Weber 1980, 123). Wo dieser „Legitimationsglaube“ fehlt, bleibt die Folgebereitschaft auf erzwingbare und kontrollierbare Verhaltensweisen beschränkt - mit dem Ergebnis, dass der Sanktionsaufwand steigt.

Verwaltungsmodernisierende Aspekte spielen im kommunitarischen Denken eine gewichtige Rolle. In den kommunitarischen Debatten wird an der Gefängnisgesellschaft USA heftig Kritik geübt. 1,6 Millionen überwiegend junge schwarze Amerikaner werden inhaftiert; das Risiko eines Schwarzen ist sieben Mal so hoch wie das eines Weißen, im Gefängnis verwahrt zu werden. Die US-Inhaftierungsrate, nach welcher Ende 1994 auf 100.000 Erwachsene 500 Häftlinge kommen (in Deutschland im Vergleichszeitraum etwa 80), bereinigt die US-Arbeitslosenstatistik.

In der kommunitaristischen Kritik am US-Gefängnissystem wird argumentiert, dass repressive Systeme antiproduktiv sind, insofern sie auf die Bewirtschaftung des vom Arbeitsmarkt ausgesperrten „,sozialen Kapitals“ verzichten. Putnam (1993) geht davon aus, 
dass das durch traditionelle Bindungen und Mitgliedschaften in Assoziationen angesammelte „soziale Kapital“ eine wesentliche Voraussetzung für die Stabilität und Modernisierung demokratischer Strukturen schafft. Ökonomische Leistungsfähigkeit einer Region hängt demnach wesentlich davon ab, wie viel ,soziales Kapital“ sich dort gebildet hat. Armutsdelinquenz verschwende hingegen systematisch Humanressourcen. Als Gegenstrategie werden jedoch nicht monetäre Verteilungssysteme diskutiert, sondern das Regulativ der Moralisierung der Bürger, welchem zugeschrieben wird, die Kosten von Überwachen und Strafen zu senken. Neben staatlichen „Zwang“ nennt der kommunitarische Pragmatiker Amitai Etzioni ein ergänzendes Regulativ, welches in der Lage sein soll, den öffentlichen Gütern Ressourcen zuzuführen: der „Bürgersinn“. Der Begriff des „Bürgersinns“ bezeichnet die „moralische Verpflichtung“"gegenüber Gemeinschaftsproblemen, die Menschen dazu veranlasst, zu dem öffentlichen Gut etwas beizutragen. Entscheidend an dieser Beurteilung des „Bürgersinns“ ist seine Nützlichkeit für die nationale Gesamtwirtschaft (Etzioni 1993)

\section{$3 \quad$ Organisierte Bürgerarbeit im europäischen Vergleich}

Der europäisch vergleichenden Studie „A New Civic Europe” zufolge sind Großbritannien und die Niederlande im Ausmaß an ehrenamtlichen Engagement in der Bevölkerung, aber auch hinsichtlich Organisation und Professionalisierung im Bereich des volunteering führend (Gaskin/Smith 1994, 95). „Make a difference” lautet das beispielhaft gewordene Programm des britischen Innenministeriums, das 1994 zur breiten Förderung ehrenamtlichen Engagements initiiert wurde. „Nach der in diesem Programm festgeschriebenen Definition des „Home Office” ist ein „Volunteer” eine Person, die persönlich Arbeit oder Dienste verrichtet (formell oder informell, mit oder ohne Vertrag) die der Gesellschaft, der Gemeinde, der Umwelt oder Personen außerhalb der eigenen Familie zugute kommen, und die dies freiwillig und ohne die Ausrichtung auf finanzielle Gewinne ausführt (Kendall/Knapp 1996, 13). In Großbritannien appellierte man in der 1995 eröffneten Kampagne „Think Volunteering” an Behörden und Ministerien, in ihrem jeweiligen Zuständigkeitsbereich die stärkere Beteiligung von Freiwilligen zu fördern. Das im gleichen Jahr gegründete „Volunteer Centre” entwickelt seither mit Firmen eine Infrastruktur für Kooperationen zwischen Wirtschaftsunternehmen und Wohlfahrtsverbänden.

Über 3 Millionen Menschen - das sind 38\% der Bevölkerung - dienen in den Niederlanden der Wohlfahrt des Gemeinwesens. 1994 errechnete das niederländische „Zentrum für Freiwilligenarbeit” Politikern und der Öffentlichkeit vor, dass umgerechnet annähernd 14,6 Milliarden Euro aufgebracht werden müssten, um diese ehrenamtlich erbrachten Leistungen marktüblich zu vergüten. Ihr Wert entspricht demnach etwa $8 \%$ des niederländischen Bruttosozialprodukts (Social and Cultural Report 1997). In den letzten Jahren sind in mehreren europäischen Industrienationen Einrichtungen entstanden, die ehrenamtliche Mitglieder rekrutieren und beraten sollen. Herausragende Beispiele dafür sind die „Volunteer Centres” in den USA und Großbritannien, die „Volunteer Bureaux” in Dänemark oder die „Freiwilligen-Zentren” in den Niederlanden. Sie bieten Informationen über ehrenamtliche Tätigkeiten an, vermitteln zwischen den „Anbietern” und den „Nachfragern”, organisieren Fortbildungen und leisten Öffentlichkeits-und Lobbyarbeit.

In zahlreichen Studien werden im „Dritten Sektor“ unausgeschöpfte Potentiale von Beschäftigung empirisch erhoben, mit dem Ziel, Regierungswissen hervorzubringen, das 
Know-how bereithält, um Massenerwerbslosigkeit in ihren Auswirkungen durch die Ausweitung des „Dritten Sektors“"zu mindern. Auch in der deutschen Debatte begleitet mittlerweile die moralische Erbauungsliteratur Schwärmereien vom gesamtwirtschaftlichen Nutzen der Gratisarbeit und von der Bewirtschaftung „,brachliegenden“ Humankapitals: „Es gibt ein gewaltiges, mehrere Millionen Menschen umfassendes Engagementpotential“ (Klages 1999, 161). Übereinstimmend setzen sämtliche Begriffsdefinitionen zivilgesellschaftlicher Arbeit unbezahlte Tätigkeiten von BürgerInnen (mit Ausnahme der „Bürgergeld“-Konzeption Ulrich Becks) voraus, die als ,,soziales Kapital“(Beck 2000, 153; Heinze/Strünck 2000, 171 216), als „Engagementpotential“ (Klages 2000, 151-170) oder als „,bürgerschaftliche Ressource“ (Etzioni 1993, 116) bei der Erstellung öffentlicher Leistungen, bei Entscheidungen über infrastrukturelle Prioritäten u. a. im Zusammenhang der Debatten um Verwaltungsmodernisierung stärker genutzt werden sollen. In Deutschland werden von Seite der Regierungen auf der Ebene von Bund und Land in den letzten Jahren empirische Studien zur Wertschöpfung der Humankapitalressourcen im Bereich ehrenamtlicher Arbeit forciert (Kommission für Zukunftsfragen 1997; Maier 1996): „Noch vor einiger Zeit wäre es undenkbar gewesen, dass von offizieller Seite die Wertschöpfung, die in einzelnen Bereichen des freiwilligen Engagements geleistet wird, in Mark und Pfennig umgerechnet wird. Jetzt findet man hierzu allenthalben Ansätze, wobei sich jeweils stolze Multi-Millionensummen und insgesamt, über alle Engagementbereiche hinweg gerechnet, Milliardensummen ergeben." (Klages 2000, 151)

Hinsichtlich der Strategien der zukünftigen Engagementförderung moniert der Verwaltungswissenschafter Helmut Klages in seinem Aufsatz „Engagement und Engagementpotential in Deutschland" ein Leitbild für BürgerInnen, welches verdeutlichen soll, dass die Bereitschaft für ehrenamtliches Engagement ,nicht mehr als eine rein private Angelegenheit betrachtet werden kann“(Klages 1999, 169). In den westlichen Bundesländern Deutschlands war 1994 annähernd ein Drittel der Bevölkerung (ca. 16 Millionen) ehrenamtlich engagiert.

In der Wirtschaft boomt das social sponsoring. So errichtete beispielsweise die amerikanische Firma Timberland in den Niederlanden ein Servicetelefon, über das landesweit Informationen über ehrenamtlichen Versicherungsschutz und Ausbildung etc. abgerufen werden können (Tijdschrift voor de Sociale Sector 1995). IBM erhebt die Sozialkompetenz zum Träger seiner Unternehmenskultur und stellt seit einigen Jahren auch in Deutschland den MitarbeiterInnen bezahlte „Sozialzeit” zur Verfügung, in der sie sich in bestimmten Projekten für behinderte Menschen engagieren können. In ihrer - ebenfalls von Beck herausgegebenen - Studie „Die Verzinsung des sozialen Kapitals“ sprechen sich Rolf Heinze und Christoph Strünck für eine politisch organisierte Erwartungshaltung der Gemeinschaft gegenüber den BürgerInnen aus: „Nur wenn ein Teil der Umwelt die Ziele der individuellen Tätigkeit als nützlich erachtet und der Prozess selbst dazu eine für rational gehaltene Produktivität aufweist, ist die Beschreibung als 'Arbeit' - nicht als 'Erwerbsarbeit' - gerechtfertigt.“ (Heinze/Strünck 2000, 181). Die gesellschaftspolitische Bewertung der Ressource „Gratisarbeit“ als „Gebrauchswert für die Umwelt“ insistiert jedoch darauf, dass freiwilliges Engagement nicht primär einem subsistenzwirtschaftlichen Zweck dient, sondern im weiteren Sinne Kollektivgüter produziert (Heinze/Strünck 2000, 181f.).

Die 1997 der Zukunftskommission für Bayern und Sachsen unter dem Titel „Erwerbstätigkeit und Arbeitslosigkeit in Deutschland. Entwicklung, Ursachen und Maßnahmen“ vorgelegte Studie widerlegt nach Auswertung der Daten des sozio-ökonomischen Panels die „Eurovol-Untersuchung“, nach der das Engagement der „Freiwilligen“ in Deutschland im 
europäischen Vergleich unter dem Durchschnitt ausgebildet ist (Gaskin/Smith 1995). Seit 1985 ist der Anteil der ehrenamtlich Aktiven um 5 Prozent gestiegen und fast ein Drittel der deutschen Bevölkerung engagieren sich in einer ehrenamtlichen Tätigkeit. Jeder Fünfte neu geschaffene Arbeitsplatz entstand in den letzten Jahren im Non-profit-Sektor. (Heinze/ Keupp 1997, 27). Bei den sozialen Diensten kommt ein ehrenamtlicher Mitarbeiter auf neun bezahlte Angestellte. Auf dem Gebiet von Freizeit und Kultur hingegen stehen sechs „Freiwilligen“ einem bezahlten Mitarbeiter gegenüber. Die politisch oft beschworenen Bewegungen der Selbsthilfegruppen und Bürgerinitiativen machen nach Anheier u.a. (1997) nur einen kleinen Teil des dritten Sektors aus. Mächtig seien nach wie vor die freien Wohlfahrtsverbände wie die katholische Caritas, die SPD-nahe Arbeiterwohlfahrt, die evangelische Diakonie, das Deutsche Rote Kreuz und der Deutsche Paritätische Wohlfahrtsverband. Sie profitieren am meisten am traditionellen Subsidiaritätsprinzip. Nach diesem Prinzip delegiert der Staat soziale Dienstleistungen möglichst an Non-Profit-Organisationen und unterstützt diese finanziell bei ihren Aufgaben. Nach der Meinung von Soziologen, die sich dem Thema zugewandt haben, seien die bestehenden Organisationen jedoch zu unbeweglich.

In ihrem Beitrag „Die Verzinsung des sozialen Kapitals“im Rahmen des von Ulrich Beck herausgegebenen Sammelbandes „Die Zukunft von Arbeit und Demokratie“ drängen die Autoren Rolf Heinze und Christoph Strünck auf eine intensive Nutzung des „Engagementpotentials“. Grundlage für eine möglichst allgemeine Diffusion von Gratisarbeit ist die Koppelung der freiwilligen Tätigkeit an eigene Erfahrungen und Fähigkeiten“ (Heinze/ Strünck 2000, 172). Durch die Individualisierung des Ehrenamtes soll keiner ausgeschlossen werden und einerseits jeder als potentieller Normadressat und andererseits jeder als zuvor brachliegendes Humankapital bewirtschaftet werden können. Die zentrale Frage des organisierten Bürgerengagements ist: wie kann eine möglichst große Anzahl von BürgerInnen möglichst nutzbringend für die Gesamtwirtschaft mobilisiert und eingesetzt werden? Um das Potential optimal auszuschöpfen, darf an den traditionellen Milieus und Sozialverbänden nicht Halt gemacht werden. Plädiert wird für die Vereinfachung und Vereinheitlichung des Vereinsrechtes sowie mehr Transparenz im Steuer- und Gemeinnützigkeitsrecht. Der Dritte Sektor sollte politisch stärker anerkannt, zugleich aber von der Staatsfinanzierung unabhängiger werden. Stattdessen sollte die private Finanzierung, unter anderem über Sponsoring und Fundraising stärker ausgebaut werden. Das social marketing, die Kunst, Menschen für Menschen zu gewinnen, soll weniger das ,Stigma” von non-profit, als das von social-profit nach außen tragen (Schaaf-Derichs 1997, 24). Wurde in den 80ern im Widerstreit zwischen Liberalismus und Kommunitarismus das nur auf seinen eigenen Vorteil bedachte wirtschaftsliberale Subjekt in einem unüberwindbaren Gegensatz zum gemeinnützigen, altruistischen Bürger gedacht, so werden in den 90ern diese beiden Positionen verschmolzen. Dieses neue Image unbezahlter Arbeit mit moralischem, ,benefit" soll eine Klientel mobilisieren, die unter der ,sozialen Kälte“ leiden und soziale Bedürfnisse nach „Einbindung, erfüllter Freizeit oder neuen Erfahrungen“ (Strümpel/Pleschberger/Riedel 1999, 224) befriedigen wollen.

An Anreizen zum Eigennutzen darf es also nicht fehlen, die individuell abgestimmte social wellness bildet in der neuen Rekrutierung Freiwilliger das Kernstück des professionellen volunteering. So ordiniert beispielsweise die Münchner Agentur „Tatendrang” mit ihrem Slogan „Spenden Sie Zeit statt Geld” das Kurmittel der Freude des Freude-Machens, um hervorzuheben, dass das Ehrenamt auch eine ,,persönliche Bereicherung und eine besondere Form des solidarischen Zusammenlebens” sein kann. In Berlin orientiert sich der, ,Treffpunkt 
Hilfsbereitschaft” und sein Beratungskonzept am „Modell des Reisebüros”: „Man stellt sich vor, dass Interessierte mit ihrem Traum vom Abenteuer in fremde Sozialwelten in die Beratungsstelle kommen. So wie ein Reisebüro dazu da ist, Urlaubsträume zu erfüllen, besteht die Aufgabe des Treffpunkts darin, seinen Kunden Angebote zu machen, wo und wie sich ihre Vorstellungen verwirklichen lassen" (Olk u.a. 1992, 27). So wird schließlich auch das „Ehrenamt” im Milieushopping seiner nützlichen Konsumtion zugeführt - in der Warenform des „come together”. Dies entspricht einem neoklassischen Standpunkt: der Dienst am Mitmenschen und Orientierung an moralischen Werten wird in einem gekoppelten Nutzen verknüpft. Dieser Ansatz des „Individualismus des selbstbestimmten Engagements“ (Beck 2000, 151) erklärt Lust und Eigennutzen wie auch das entgegengesetzte Verhalten Sorge um andere und um die Gemeinschaft - mittels der gleichen eigennutzorientierten Begriffe zu bestimmen.

Bürgerarbeit und andere mit ehrenamtlichem Engagement und Gemeinsinn in Zusammenhang gebrachten Tätigkeiten werden scheinbar geschlechtsneutral angeboten. Wie zahlreiche internationale Studien erhoben haben (vgl. exemplarisch: Notz 1986, 1987, 1989; Reihs 1997), weist insbesondere der Bereich der Gratisarbeit hinsichtlich der geschlechtsspezifischen Arbeitsteilung eine hierarchisch ausgeprägte Tradition auf, die den gesamten Bereich des volunteering kennzeichnet. Die Statistik der Bundesarbeitsgemeinschaft der Wohlfahrtsverbände weist Jahr für Jahr 1,5 Millionen ehrenamtliche Mitarbeiter aus. Zwei Drittel davon sind Frauen. Dort wo es um unmittelbar mit Sorge und Pflege verbundene Arbeiten geht, sind 80\% Frauen tätig. Frauen sind mehrheitlich in der Basisarbeit, Männer mehrheitlich in leitenden Gremien und Ehrenämtern tätig.

Während die Vorstände der Wohlfahrtsverbände, die Aufsichtsräten kultureller, gesellschaftlicher, politischer, wirtschaftlicher, wissenschaftlicher und kirchlicher Gremien überwiegend von männlichen Funktionären besetzt sind, ist die ehrenamtliche Basisarbeit im sozialen Bereich als unbezahlte, fürsorgerische Arbeit ohne Schutz und ohne Sicherung der Arbeitsbedingungen den Frauen vorbehalten.

Im (verschwiegenen) Zentrum der neuen Konzepte der „Gratisarbeit“ steht die gesamtwirtschaftliche Strategie der Senkung der Staatsausgaben. An einer Aufhebung der sozialen und der geschlechtsspezifischen Ungleichheit im Produktionsprozess ist jedoch kaum jemand interessiert. Erhalten bleiben soll stets eine Konstante: die Medikation der bestehenden Sozialordnung.

\section{4 „Belohnung“6 im Niedriglohnsektor}

Einer der Schlüsselbegriffe im Diskurs gemeinwohlorientierter Arbeiten im Dritten Sektor ist der Begriff der „Bürgerarbeit“ - am prononciertesten formuliert von Ulrich Beck im Rahmen des Zukunftsberichtes der Bayrisch-Sächsischen Zukunftskommission (Kommission für Zukunftsfragen 1997). Für die von der Erwerbsarbeit Ausgeschlossenen empfiehlt er der bayrischen Landesregierung die „Bürgerarbeit”, die von „Arbeitslosen und Sozialhilfeempfängern, Jugendlichen neben und nach der Berufsausbildung, Müttern nach der Erziehungsphase, älteren Menschen im Übergang in den Rentenstand und Teilzeiterwerbstätigen" (Kommission 1997, 3) geleistet werden könne.

Beck beschreibt „Bürgerarbeit“ als projektgebundene Tätigkeit, die kooperativ, selbst organisiert und zeitlich begrenzt ist (Beck 1999b, 133), für die nur derjenige „Bürgergeld“ 
erhält, der über kein ausreichendes Einkommen verfügt und also auf eine Sozialunterstützung existenziell angewiesen ist (Beck 1999b, 133). „Bürgerarbeit wird (...) nicht entlohnt, aber belohnt und auf diese Weise sozial anerkannt und aufgewertet." (Beck 1999b, 128); immateriell wird „Bürgerarbeit“ durch „Favour Credits“, Ehrungen, Qualifikationen und Anerkennung von Rentenansprüchen und Sozialzeiten (Heinze/Keupp 1997, 126) aufgewertet. Materiell gäbe es für diese Tätigkeiten lediglich das „Bürgergeld“, eine „Belohnung“ in Höhe des Sozialhilfe- oder Arbeitslosenhilfesatzes (Beck 2000, 133).

Durch die Erschließung „,nicht-marktgängiger, gemeinwohlorientierter Tätigkeitsfelder“ (Kommission 1997, 146) soll die Zahl der Erwerbslosen verringert werden, insofern gemeinnützig Tätige dem Arbeitsmarkt nicht zur Verfügung stehen und aus der Arbeitslosenstatistik entfallen. Unter dieser Prämisse soll „Bürgerarbeit“ als veritables Konzept zur Reduzierung der Arbeitslosigkeit fungieren. Nichtmarktgängige und gemeinwohlorientierte Tätigkeitsfelder sollen zu einem neuen attraktiven Zentrum gesellschaftlicher Aktivität gebündelt und mit dem Sachverhalt anhaltend hoher Massenarbeitslosigkeit als Ausdruck der Krise der Erwerbsgesellschaft konfrontiert werden. Daraus wird voreilig der Schluss gezogen, die kapitalwirtschaftlich orientierten Industrienationen befänden sichim Allgemeinen am „Ende“ der Lohnarbeit und am Anfang der „Tätigkeitsgesellschaft“. Ulrich Beck und viele andere Autoren im Meanstream in der aktuellen Diskussion um gesellschaftspolitische Reformen entwickeln ihre Optionenberichte der „Neuen Arbeitsformen“ ausgehend von dem Befund, dass Vollbeschäftigung und Lohnerwerbsarbeit innerhalb der gesellschaftstheoretischen Analyse „endgültig“ den Status einer Schlüsselkategorie verloren haben. Beck konstatiert eine sich zu „Ende neigenden Erwerbsgesellschaft“ (Beck 1999b, 9), welche kraft seiner Behauptung existiert, leitet daraus ein gesellschaftspolitisches Problem der ,Unsicherheit“ ab, dessen „Bedrohung“ er zum Anlass nimmt, seinem eigenen Diskurs einen notwendigen Handlungsbedarf zu unterstellen. .

Folgt man der Annahme eines ,globalen Sachzwanges“ schwindender Lohnarbeit, dann ergeben sich zwangsweise in Reaktion darauf nur noch „Anpassungsstrategien“, wie sie etwa der erwähnte bayrisch-sächsische Zukunftsbericht vorsieht, darin die „Fixierung auf Erwerbsarbeit" mit dafür verantwortlich gemacht wird, dass der Arbeitsmarkt nicht flexibel genug auf Arbeitslosigkeit reagieren kann. Die von Meinhard Miegel geleitete „Kommission für Zukunftsfragen“" spricht hier von einem „Substanzverfall abhängiger Erwerbsarbeit“ (Süddeutsche Zeitung vom 23.10.1996). In sozialtechnischer Hinsicht geht es folglich darum, bezahlte Erwerbs- und Berufsarbeit in Beschäftigung zum Nulltarif zu transformieren oder qua „Bürgerarbeit“ den nationalen Niedriglohnsektor zu konsolidieren. Gemäß Ulrich Becks Ausführungen soll „Bürgerarbeit“ nicht bloß die Defizite öffentlicher Infrastruktur kompensieren, sondern gebunden an eine „Belohnung“, dem Bürgergeld, soll das Segment der Niedrig-Löhne stabilisiert und längerfristig gesichert werden, wie es Beck explizit als einer der zentralen Schwerpunkte der Bürgerarbeit ausweist: „Ermöglichung eines Niedriglohnsektors (...) im Bereich der Globalisisierungsverlierer“(Beck 1999b, 146). Professionalisierte „Bürgerarbeit“ wird Arbeitslosen ohne tarifliche Bezahlung und entsprechende Rechte des Arbeitnehmers übertragen. Als Bemessungsgrundlage für das „Bürgergeld“ gilt die ,politische Leistung“(Beck 1999b, 146). Mit der Anknüpfung der ,,ehrenamtlichen Tätigkeit“ an den staatsbürgerlichen Rechtsstatus, den „Bürgerstatus“ (Beck 1999b, 146), ist ausländischen ArbeitsmigrantInnen der Zugang zum „Bürgergeld“verwehrt. Damit einhergehend kann beispielsweise eine Verdrängungskonkurrenz am Arbeitsmarkt der mit Sorge und Pflege verbundenen Arbeiten erwartet werden, die auf der Zunahme von inländi- 
schen „BürgerarbeiterInnen“ beruht und zum Abbau von ausländischen ArbeitsmigrantInnen führen soll (vgl. Notz 1986, 54ff).

Das mit dem Begriff des moralischen Lohnes verknüpfte Konzept des „Bürgergeldes“ suspendiert das Konkurrenzprinzip der objektiven Bestimmbarkeit des Leistungslohnes (Prinzip der Rationalisierung). Beck nennt den Individualismus des selbstbestimmten Engagements ,zutiefst moralisch“ (Beck 1999b, 153). Der Arbeitsbegriff Ulrich Becks ist einem Postulate-Kanon eingebettet und somit nicht deskriptiv, sondern präskriptiv orientiert. Die Kluft zwischen Existenzlohn und Nulltarif soll durch die Bereitwilligkeit zur persönlichen Investition geschlossen werden. Dieses Amalgam der „Spaßarbeit” - als wäre Lohnarbeit eine naturgegebene Frustration - verstellt jedoch den Blick auf die politische Ökonomie der unbezahlten Arbeit, denn während derÖkonom den Wert der Arbeitskraft als gesellschaftlich notwendige verrechnet, kann der persönlich engagierte und tolerante Sozialarbeiter, der den sozial benachteiligten „Nachbarn“, „Minderheiten“ und „Ethnien“ hilft, bloß seine partikuläre moralische „Belohnung“erwarten. An Stelle des materiellen Lohns rückt die moralische Belohnung. Vermögensbildend ist die moralische Belohnung aber ausschließlich durch soziale Anerkennung einzelner Betroffener. Es wird dadurch nicht nur der Aspekt des von der „Bürgerarbeit“" erwirtschafteten Sozialprodukts ausgeblendet, es sollen gleichzeitig Sozialhilfeempfänger abgebaut werden. Durch die moralische Gabebeziehung im „Bürgerarbeit“ verlieren beide, der Gebende wie der Nehmende, die berechtigten Ansprüche auf Teilhabe des von ihnen erwirtschafteten Sozialproduktes.

\section{Das republikanische Ideal der „Bürgerarbeit““}

Das Konzept der „Bürgerarbeit“ soll neben arbeitsmarkt- und sozialpolitischen Bezügen darüber hinaus durch die Koppelung von Bürgerarbeit und Ehrenamt zur Reaktivierung der gefährdeten „Seele der Demokratie“ (Beck 2000, 416f) beitragen. Darin firmiert „Bürgerarbeit“ als Instrument des republikanischen Ideals der „selbsttätigen Bürgergesellschaft, welche ihre eigenen Angelegenheiten aktiv in die Hände nimmt" (Beck 1999b,146).

In Berücksichtigung Marcel Mauss` Untersuchung der Gabenmoral bezeichnen wir das ehrenamtliche Engagement als „Produkt eines moralischen Begriffs der Gabe und des Reichtums einerseits und des Begriffs des Opfers andererseits” (Mauss 1989, 35). Sozialpsychologischen Prädikaten der bürgerlichen Freiheit wie „Eigensinn“, Eigeninitiative“, ,,Spontanität“, „,Selbstentfaltung“, „Buntheit“”, „Selbstverwirklichung“ oder „Authentizität“u.a. wird das Gute-an-sich zugrundegelegt, das schließlich als moralischer Wert dem Allgemeinbegriff der Arbeit subsumiert wird. Diese Auffassung von einem Bürger als einem freien, mit dem Bedürfnis nach „moralischem Individualismus“ (Beck 2000, 153), ausgestatteten Subjekt verwandelt schließlich dieses moralische Attribut in ein moralisches Urteil, das den Menschen als eine anthropologische Konstante begleitet.

Die produktive Ordnung der Demokratie gründet Beck auf den menschlichen Affekt der „Arbeits“- und „Leistungsfreude“. Dieser „Bürgersinn“ der Arbeit wird als conditia humana gesetzt, wodurch der Bürger in der Äußerung seiner menschlichen Natur faktisch eindeutig bestimmt erscheint. Determiniert durch einen metaphysischen Menschenbegriff haben die Bürger von sich aus einen Bedürfnis nach „Anerkennung“ und „Autonomie“, wodurch sie in die Abhängigkeit der Befriedigung ihrer subjektiven Bedürfnisse versetzt werden, die als ursprünglich gegeben vorausgesetzt sind. Insofern die Motivation zur Arbeit hauptsächlich 
von der Persönlichkeit und ihrer Bereitwilligkeit hervorgebracht wird, ist die Sozialtechnologie von der von ihr selbst als repressiv vorausgesetzten Agenda dirigistischer Einmischung befreit. Dadurch kann sich die Sozialtechnologie, wie sie Ulrich Beck formuliert, auf die Fragestellung zurückziehen, unter welchen Bedingungen „Leistungsfreude“ und „Einsatzbereitschaft" der Bürger, unbezahlt oder mehr zu arbeiten, eintreten.

\section{Abschliessende Zusammenfassung}

Das Ziel dieses Beitrags war es, die sozialtechnische Komplementarität des diskursiven Allianzdispositivs Morallehre - Politische Ökonomie betreffend den Gegenstand der „Bürgerarbeit" herauszuarbeiten. Es wurde untersucht, inwiefern beide Diskurse eine arbeitsteilige Allianz bilden, um zivilgesellschaftliche Arbeit regierbar und administrierbar zu machen. Der pädagogische Diskurs der moralischen Disziplin erstellt gegenüber Normadressaten normative Regeln gemeinwohlorientierten Engagements, während der Diskurs der Politischen Ökonomie gegenüber Regierungsexperten regulative Modelle gesamtwirtschaftlicher Wertschöpfung der Gratisarbeit ableitet. Die Komplementarität von Moral und Ökonomie erweist sich in der Gleichsetzung des Begriffes der Wirksamkeit im Sinne technischer Durchführbarkeit und des Begriffes der Zulässigkeit im Sinne normativer Bewertbarkeit.

Die Kritik der Moralisierung der zivilgesellschaftlichen Arbeit, die vorrangig auf den Konzepten psychologischer Lebensweltlichkeit, sozialer Typenlehre und individueller Zurechnungsfähigkeit beruht, ging von folgender Problemstellung aus: Im Allgemeinen wird „zivilgesellschaftliche Arbeit“(Ellis/Noyes 1990,13) als eine allgemein menschliche Tätigkeit definiert, die einem „menschlichen Bedürfnis” entspringe und - ohne monetären Profit - sozial anerkannt wird. Doch ist mit der libidinösen Bindung an die Arbeit bereits der Sprung ins Reich der Freiheit geglückt, darin die Disziplinen aufgehoben sind? Ist aber das menschliche Bedürfnis nicht ,ein sorgfältig gepflegtes, kalkuliertes und ausgenutztes politisches Instrument?“ (Foucault 1977, 37).

Die Kritik der Politischen Ökonomie der zivilgesellschaftlichen Arbeit fokussierte folgende Problemstellung: In dem umfassenden Projekt eines moralisch fundierten Utilitarismus, das in Deutschland am prononciertesten von Ulrich Becks Modell der „Bürgerarbeit“ forciert wird, sollen „Eigeninteresse“ und „Gemeinwohl“ versöhnt werden. Das Modell der „Bürgerarbeit“ sieht vor, gemeinnützige Arbeiten der „Bürger“ monetär zu „,belohnen“. Aus der allgemeinen Moralisierung soll die gesamtwirtschaftlich relevante Wertschöpfung abgeleitet werden. Darüberhinaus soll mit diesem finanziellen Anreizsystem der Widerstreit zwischen Liberalismus und Kommunitarismus und ihrer Dichotomie „Eigennutzen-Gemeinnutzen“ aufgehoben werden. Wenn moralisches Handeln jedoch unter dem instrumentellen Aspekt der „Bewältigung der Finanzkrise der öffentlichen Haushalte“ (Klages 2000, 151) positiv beurteilbar werden soll, dann wird das normative Postulat der „Freiwilligkeit“ und „Autonomie“" zivilgesellschaftlicher Arbeit problematisch und gleichwohl der Befreiungsdiskurs von der Entmündigung durch Lohnabhängigkeit, der im Diktum gipfelt, dass Arbeit „frei“ machen soll, aporetisch. 


\section{Literatur}

Anheier, Helmut K. u. a. (1997): Der Dritte Sektor in Deutschland. Berlin

Artner, Margarethe u.a. (1995): Ehrenamt zwischen Anspruch und Wirklichkeit. Wien

Barber, Benjamin (1994): Starke Demokratie. Über die Teilhabe am Politischen. Hamburg

Beck, Ulrich (1997): Was heißt Globalisierung? Irrtümer des Globalismus - Antworten auf Globalisierung. Frankfurt/M.

Beck, Ulrich (1999a): Kinder der Freiheit: Wider das Lamento über den Werteverfall; in: Ulrich Beck (Hg.): Kinder der Freiheit. Frankfurt/M., 9-33

Beck, Ulrich (1999b): Schöne neue Arbeitswelt. Vision: Weltbürgerschaft. Frankfurt/M.

Beck, Ulrich (2000): Wohin führt der Weg, der mit dem Ende der Vollbeschäftigungsgesellschaft beginnt?; in: Ulrich Beck (Hg.): Die Zukunft von Arbeit und Demokratie. Frankfurt/M., 7-66

Belah, Robert u.a. (1985): Habits of the Heart: Individualism and Commitment in American Life. Berkely

Bourdieu, Pierre (1990): Was heißt sprechen? Die Ökonomie des sprachlichen Tausches. Wien

Coleman, James (1988): Social Capital in the Creation of Human Capital; in: American Journal of Sociology 94 (Supplement), 95-120

Demirovic, Alex (1997): Demokratie und Herrschaft. Aspekte kritischer Gesellschaftstheorie. Münster

Durkheim, Emile (1991): Physik der Sitten und des Rechts. Vorlesungen zur Soziologie der Moral. Frankfurt/M.

Ellis, Susan, Katherine Noyes (1990): By The People. A History of Americans as Volunteers. San Francisco

Etzioni, Amitai (1993): Über den Eigennutz hinaus; in: Josef Wieland (Hg.): Wirtschaftsethik und Theorie der Gesellschaft. Frankfurt/M., 109-133

Etzioni, Amitai (1995): Die Entdeckung des Gemeinwesens: Ansprüche, Verantwortlichkeiten und das Programm des Kommunitarismus. Stuttgart (Das Kommunitaristische Programm, 282-299)

Etzioni, Amitai (1996): Die faire Gesellschaft. Jenseits von Sozialismus und Kapitalismus. Frankfurt/ M.

Foucault, Michel (1977): Überwachen und Strafen. Die Geburt des Gefängnisses. Frankfurt/M.

Gaskin, Katherine, Justin Davis Smith (1995): A New Civic Europe? A Study of the Extent and Role of Volunteering. London

Gorz, André (2000): Arbeit zwischen Misere und Utopie. Frankfurt/M.

Heinze, Rolf, Heiner Keupp (1997): Gesellschaftliche Bedeutung von Tätigkeiten außerhalb der Erwerbsarbeit. Gutachten für die Kommission für Zukunftsfragen der Freistaaten Bayern und Sachsen. Bochum/München

Heinze, Rolf, Christoph Strünck (2000): Die Verzinsung des sozialen Kapitals. Freiwilliges Engagement im Strukturwandel; in: Ulrich Beck (Hg.): Die Zukunft von Arbeit und Demokratie. Frankfurt/ M., 171-216

Helliwell, John F. (1996): Do Borders Matter for Social Capital? Economic Growth and Civic Culture in U.S. States and Canadian Provinces. National Bureau of Economic Research, Working Paper 5863. Cambridge/Mass.

Kelsen, Hans (1960): Reine Rechtslehre. Wien

Kendall, Jeremy, Martin Knapp (1996): The Voluntary Sector in the UK. Manchester

Kramer, Ralph (1981): Voluntary Agencies in the Welfare State. Los Angeles

Kommission für Zukunftsfragen der Freistaaten Bayern und Sachsen (1997). Erwerbstätigkeit und Arbeitslosigkeit in Deutschland. Entwicklung, Ursachen und Maßnahmen. Teil 3: Maßnahmen zur Verbesserung der Beschäftigungslage. Bonn

Mauss, Marcel (1989): Die Gabe, Form und Funktion des Austauschs in archaischen Gesellschaften, in: Marcel Mauss: Soziologie und Anthropologie 2, Frankfurt/M., 11-148

Meier, Bernd (1996): Sozialkapital in Deutschland - eine empirische Skizze; in: Institut der deutschen Wirtschaft (Hg.): Beiträge zur Wirtschafts- und Sozialpolitik 6, 231 
Nietzsche, Friedrich (1994): Zur Genealogie der Moral; in: Karl Schlechta (Hg.): Nietzsche. Werke in drei Bänden. München, 2, 876

Notz, Gisela (1986): Zur Gestaltung ehrenamtlicher sozialer Arbeit. Forschungsbericht. FriedrichEbert-Stiftung. Bonn

Notz, Gisela (1987): Arbeit ohne Geld und Ehre? Zur Gestaltung ehrenamtlicher sozialer Arbeit. Opladen

Notz, Gisela (1989): Frauen im sozialen Ehrenamt. Ausgewählte Handlungsfelder: Rahmenbedingungen und Optionen. Freiburg

Olk, Thomas (1998): Der Sozialstaat als Herausforderung. Opladen

Olk, Thomas u.a. (1992): Von der traditionellen Sozialpolitik zur neuen Wohlfahrtskultur? Freiwilliges soziales Engagement und lokale Infrastruktur. ILS-Schriften 64, 27-43

Putnam, Robert (1994): Making Democracy Work. Princeton

Reihs, Sigrid (1995): Im Schatten von Freiheit und Erfüllung. Ehrenamtliche Arbeit in Bayern. Bochum Rifkin, Jeremy (1997): Das Ende der Arbeit und ihre Zukunft. Frankfurt/M./New York

Roth, Roland (1995): Kommunitaristische Sozialpolitik. Anmerkungen zur aktuellen Debatte über Professionalität und Ehrenamt in der Sozialpolitik; in: Forschungsjournal Neue Soziale Bewegungen 8 (3), 44-53

Schaaf-Derichs, Christian (1997): Eine Agentur für "Social Profit"; in: sozialmagazin 3, 24

Sennett, Richard (1998): Der flexible Mensch. Die Kultur des neuen Kapitalismus. Berlin Verlag

Social and Cultural Report 1996 (1997): The Netherlands. Rijswijk

Strümpel, Charlotte, Sabine Pleschberger, Birgit Riedel (1999): Freiwilliges Engagement in Niederösterreich. Status quo und internationale Beispiele der Förderung. Europäisches Zentrum für Wohlfahrtspolitik und Sozialforschung, Wien

Tijdschrift voor de Sociale Sector (1995): Vrijwilligerswerk. Utrecht

Western, Bruce, Catherine Beckett (1998): Der Mythos des freien Marktes. Das Strafrecht als Institution des US-amerikanischen Arbeitsmarktes; in: Berliner Journal für Soziologie 2, 98, 159-180

Wacquant, Louis (1997): Vom wohltätigen Staat zum strafenden Staat. Über den politischen Umgang mit Armut und Elend in den USA; in: Leviathan, 1, 1-17

Weber, Max (1980): Wirtschaft und Gesellschaft. Grundriss der verstehenden Soziologie. Tübingen

Wuthnow, Robert (1991): Acts of compassion. Caring for others and helping ourselves. Princeton

Anschrift des Verfassers:

Ramón Reichert

FEDA

Forschungsgruppe Epistemologie \& Diskursanalyse

Franzengasse 5/53

A-1050 Wien

\section{Schlagwörter: Bürgerarbeit, Dritter Sektor, Neues Ehrenamt, Volunteering}

